\title{
Energy Harvesting Based on Magnetic Dispersion for Three-Phase Power System
}

\author{
Tarcisio Oliveira de Moraes Júnior, Yuri Percy Molina Rodriguez, Ewerton Cleudson de Sousa Melo, \\ Maraiza Prescila dos Santos, Cleonilson Protásio de Souza \\ Federal Institute of Paraíba - IFPB, Cajazeiras, Brazil \\ Department of Electrical Engineering, Federal University of Paraíba - UFPB, João Pessoa, Brazil \\ Email: tarcisiocz@gmail.com, \{molina.rodriguez\} \{protasio\}\{maraiza.santos\}\{ewerton\}@cear.ufpb.br
}

Received 2013

\begin{abstract}
This paper presents a comparative study on Magnetic-Dispersion based Energy Harvesting Systems (MD-EHS) on electrical conductors that supply power for a three-phase AC motor. It introduces two MD-EHS which are based on magnetic cores of different material, named, nanocrystalline, ferrite and iron powder. The first one consists of harvesting energy from magnetic flux through three symmetrical magnetic cores installed on each power conductors of a three-phase AC motor. The second one consists of a single magnetic core for harvesting energy from magnetic flux of only one of these conductors. Both ones have an AC/DC converter and a variable resistor based load. Experimental results have agreed with the theoretical analysis and show that the first proposed MD-EHS is capable of supplying 14 times more energy than the second MD-EHS, considering nanocrystalline cores and phase current of $3 \mathrm{~A}$, and 7.5 times more energy, considering ferrite cores and phase current of 9 A. Such energy can be applied to various low-power devices, especially in wireless sensor network.
\end{abstract}

Keywords: Energy Harvesting; Magnetic Dispersion; Magnetic Cores

\section{Introduction}

Energy Harvesting is the process of capturing small amounts of energy from energy sources available in environment, for instance: thermal, solar, mechanical, magnetic induction, and others, and it is specially applied in supplying energy to low-power devices particularly those from wireless sensor networks. Energy harvesting system based on magnetic induction is receiving considerable attention since it is also applicable in measuring variable in power lines.

An example of application of energy harvesting by magnetic induction can be seen in [1] in which a system composed basically of a magnetic flux device is capable of transmitting to a base station the values of temperature variations of the power line where itself is installed. In [2] is proposed a system to harvest energy from electrostatic field created between a power line and the ground. Experimental results have shown that the system can harvest $16 \mathrm{~mW}$. In [3] was studied an energy harvester based on magnetic induction on power line using a simple circuit model to validate the obtained theoretical results. As a result, $1 \mathrm{~mW}$ of power was achieved considering air-core and $6.32 \mathrm{~mW}$ considering an iron core from a magnetic field of $21.2 \mathrm{uT}$. Recently, in [4] it was stud- ied a tube shaped energy harvester from power lines where the power conditioning circuit is constraint for constant voltage. As a result, the circuit efficiency does influence the level of its output voltage. For constant output power, the voltage level of the power conditioning circuit decreases while the voltage of the transmission line increases.

In this work, it is presented a comparative study on Magnetic-Dispersion based Energy Harvesting Systems (MD-EHS) on electrical conductors that supply power for a three-phase AC motor. It was developed two MDEHS's which are based on magnetic cores of different material, named, nanocrystalline, ferrite and iron powder. The first one, called 3F-MD-EHS, consists of harvesting energy from magnetic flux through three symmetrical magnetic cores installed on each power conductors of a three-phase AC motor. The second one, called 1F-MDEHS, consists of a single magnetic core for harvesting energy from magnetic flux of only one of these conductors.

This work is organized as follows: Section II describes the essential theory of magnetic field regarding the cores specifications, Section III shows the experimental analysis, Section IV, the experimental results, and Section V, the main conclusions. 


\section{Magnetic Field Theory}

According to Ampere's law, the magnetic flux density at a given distance $r$ from a infinitely long conductor carrying an alternating current with a peak amplitude $I$ and frequency $\omega$ is given by:

$$
B=\frac{\mu_{m} I \sin (\omega t)}{2 \pi r}
$$

where $B$ is the magnetic flux density in a distance $r$ of the conductor and $\mu_{\mathrm{m}}$ is the magnetic permeability of the material between the conductor and the point $r$.

Figure 1 shows a laminated core on the power conductor in a transversal way which the proposed MDEHS's are based on. This core provides the magnetic path for the magnetic flux and consists of about 50 thin strips that are electrically separated by a thin layer of insulating material. A coin, not shown in the figure, is on the laminated core and is where the inducted voltage takes place.

Based on Figure 1, the concatenated magnetic flux, $\varphi$, across the laminated core, with sectional area $A$, is given by:

$$
\phi=\int B d A
$$

where

$$
d A=w d r
$$

Substituting (1) and (3) in (2), it is obtained:

$$
\phi_{L}=\frac{\mu_{m} I \sin (\omega t)}{2 \pi} w \int_{r_{L}}^{r_{f}} \frac{d r}{r}
$$

which can be reduced as follows:

$$
\phi_{L}=\frac{\mu_{m} I \operatorname{sen}(\omega t) w}{2 \pi} \ln \left(\frac{r_{L}+h}{r_{L}}\right)
$$

where $\phi_{L}$ : magnetic flux of the magnetic $L$-th strip of the core (where $0<L<N_{L}, N_{L}=$ number of core strips)

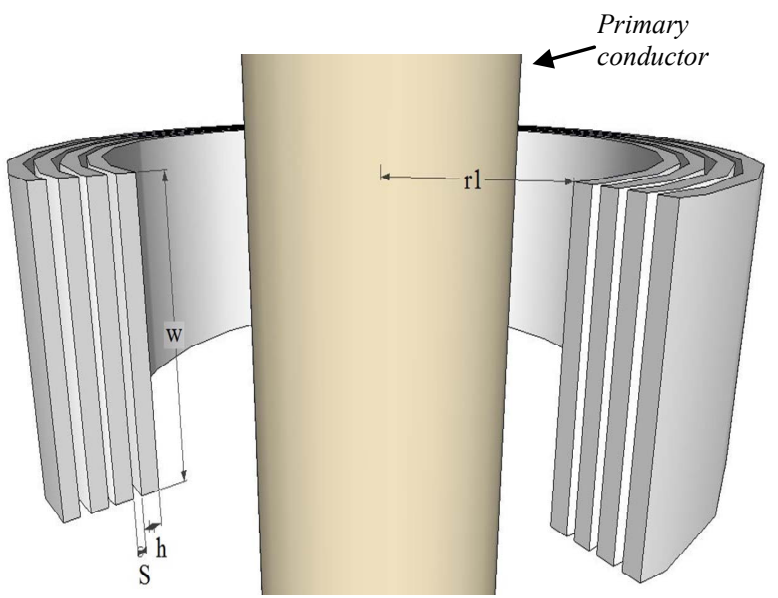

Figure 1. Laminated core on a power conductor. and $r_{L}$ is the radius of the $L$-th strip.

Concern the magnetic flux into the insulating material between the laminated core strips, it is obtained from a similar way and it is obtained:

$$
\phi_{P}=\frac{\mu_{0} I \operatorname{sen}(\omega t) w}{2 \pi} \ln \left(\frac{r_{P}+S}{r_{P}}\right)
$$

where $\varphi_{\mathrm{p}}$ : magnetic flux of the magnetic $P$-th strip of the core (where $0<P<N_{L-I}$ ) and $r_{P}$ is the radius of the $P$-th strip.

Considering now that is a coin wounds around the core with $N_{2}$ turns. In this way, the output voltage of coin terminals, according to Faraday's Law, is expressed as:

$$
V_{S}=-N_{2} \frac{d \phi_{T}}{d t}
$$

where $\phi_{T}$ is the total magnetic flux. $\phi_{T}$ can be obtained by the summation of the magnetic flux through the magnetic and insulating blades:

$$
\frac{d \phi_{T}}{d t}=\sum_{L=1}^{L=n} \frac{d \phi_{L}}{d t}+\sum_{P=1}^{P=n-1} \frac{d \phi_{P}}{d t}
$$

where:

$$
\begin{aligned}
\frac{d \phi_{L}}{d t} & =\frac{\mu_{m} I \omega \cos (\omega t) w}{2 \pi} \ln \left(\frac{r_{L}+h}{r_{L}}\right) \\
\frac{d \phi_{P}}{d t} & =\frac{\mu_{0} I \omega \cos (\omega t) w}{2 \pi} \ln \left(\frac{r_{P}+S}{r_{P}}\right)
\end{aligned}
$$

To obtain theoretical results, it was considered as parameters the core dimensions: $w, h, S$, and $r_{l}$ (internal radius), as shown in Figure 1.

\section{Experimental Study}

In order to design the proposed MD-EHS, it was used magnetic cores of three different materials: iron powder, ferrite and nanocrystalline. The first proposed MD-EHS, called 3F-MD-EHS, consists of harvesting energy from magnetic flux through three symmetrical magnetic cores installed on each power conductors of a direct start three-phase AC motor, as can be seen in Figure 2.

In Figure 3, it is shown the power conditioning circuits (PCC) for the 3F-MD-EHS and in Figure 4 it is shown for the 1F-MD-EHS.

The second proposed MD-EHS, called 1F-MD-EHS, consists of a single magnetic core for harvesting energy from magnetic flux of only one of these conductors.

It was carried out several experiments considering cores of iron powder, ferrite and nanocrystalline and load values, $R_{v}$, ranging from $10 \Omega$ up to $10 \mathrm{k} \Omega$. In all experiments, the phase current of each conductor is $3 \mathrm{~A}$ for the nanocrystalline cores, 9 A for ferrite and $12 \mathrm{~A}$ for iron powder. The main parameters of the used cores are described in Table 1. 


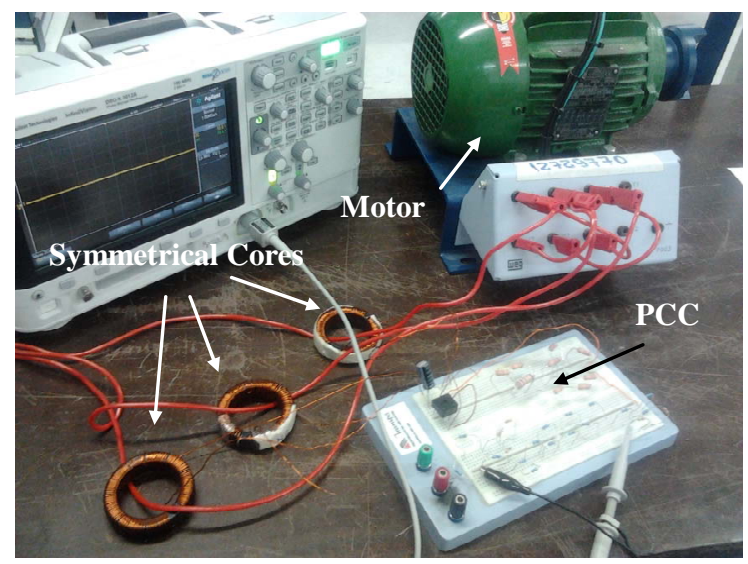

Figure 2. 3F-MD-EHS.

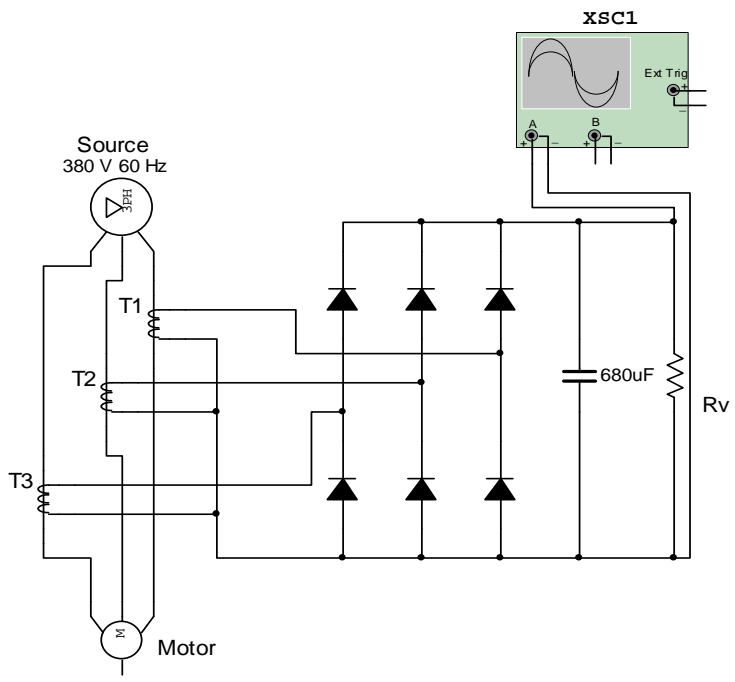

Figure 3. Power conditioning circuit of the 3F-MD-EHS.

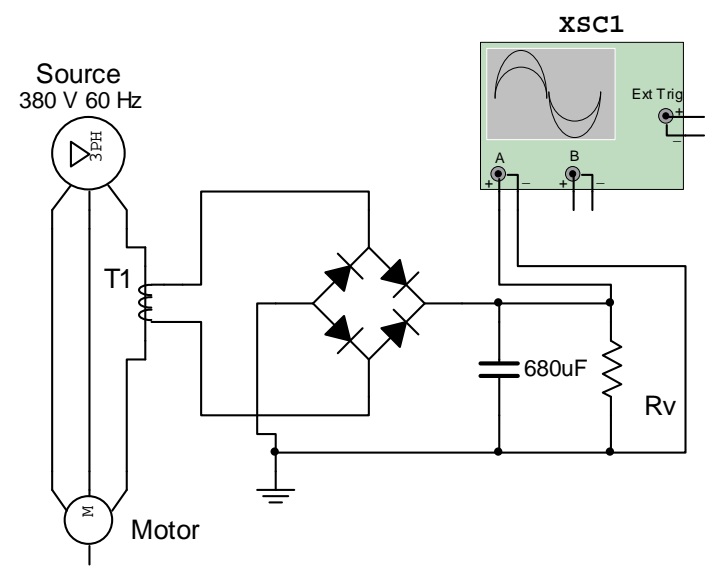

Figure 4. Power conditioning circuit of the 1F-MD-EHS.

\section{Experimental Results}

After performing experiments considering the different cores and the set of values of $R_{v}$, it was possible to obtain the maximum obtained power and the voltages values.
The best results are described in Tables 2 and $\mathbf{3}$ and the results considering the variations of $R_{v}$ can be seen in Figure 5 for the 3F-MD-EHS and Figure 6 for the 1FMD-EHS showing the power obtained for each value of the load, Rv.

Table 1. Parameters of the used cores.

\begin{tabular}{cccccc}
\hline & \multicolumn{5}{c}{ Core's Parameters } \\
\cline { 2 - 6 } Material & $\begin{array}{c}\mathrm{w} \\
{[\mathrm{mm}]}\end{array}$ & $\begin{array}{c}\mathrm{h} \\
{[\mathrm{mm}]}\end{array}$ & $\begin{array}{c}\mathrm{r} \\
{[\mathrm{mm}]}\end{array}$ & $\mathrm{S}$ & $\mu_{r}$ \\
\hline Ferrite & 8 & 4.2 & 6.85 & 0.0006 & 2300 \\
Nanocrystalline & 10.35 & 4.4 & 22.5 & 0.0006 & 1000000 \\
Iron Powder & 18 & 11.3 & 12.05 & 0.0006 & 75 \\
\hline
\end{tabular}

Table 2. Experimental results from 3F-MD-EHS.

\begin{tabular}{cccccc}
\hline \multirow{2}{*}{ Material } & \multicolumn{5}{c}{ 3F-MD-EHS } \\
\cline { 2 - 6 } & $\begin{array}{c}\mathrm{V} \\
{[\mathrm{mV}]}\end{array}$ & $\begin{array}{c}\mathrm{I} \\
{[\mathrm{mA}]}\end{array}$ & $\begin{array}{c}\mathrm{P} \\
{[\mathrm{mW}]}\end{array}$ & $\begin{array}{c}\mathrm{Rv} \\
{[\Omega]}\end{array}$ & $\begin{array}{c}\text { Ip } \\
{[\text { Arms }]}\end{array}$ \\
\hline Ferrite & 560 & 11.3 & 6.3 & 50 & 3.0 \\
Nanocrystalline & 374 & 37.4 & 14 & 10 & 9.0 \\
Iron Powder & 5.7 & 0.57 & 0.0032 & 10 & 12.0 \\
\hline
\end{tabular}

Table 3. Experimental results from 1F-MD-EHS.

\begin{tabular}{|c|c|c|c|c|c|}
\hline \multirow[b]{2}{*}{ Material } & \multicolumn{5}{|c|}{ 1F-MD-EHS } \\
\hline & $\begin{array}{c}\mathrm{V} \\
{[\mathrm{mV}]}\end{array}$ & $\begin{array}{c}\mathrm{I} \\
{[\mathrm{mA}]}\end{array}$ & $\begin{array}{c}\mathrm{P} \\
{[\mathrm{mW}]}\end{array}$ & $\begin{array}{l}\mathrm{Rv} \\
{[\Omega]}\end{array}$ & $\begin{array}{c}\text { Ip } \\
\text { [Arms] }\end{array}$ \\
\hline Ferrite & 310 & 4.6 & 1.5 & 70 & 3.0 \\
\hline Nanocrystalline & 602 & 1.5 & 0.9 & 400 & 9.0 \\
\hline Iron Powder & 18.5 & 1.85 & 0.034 & 10 & 12.0 \\
\hline
\end{tabular}
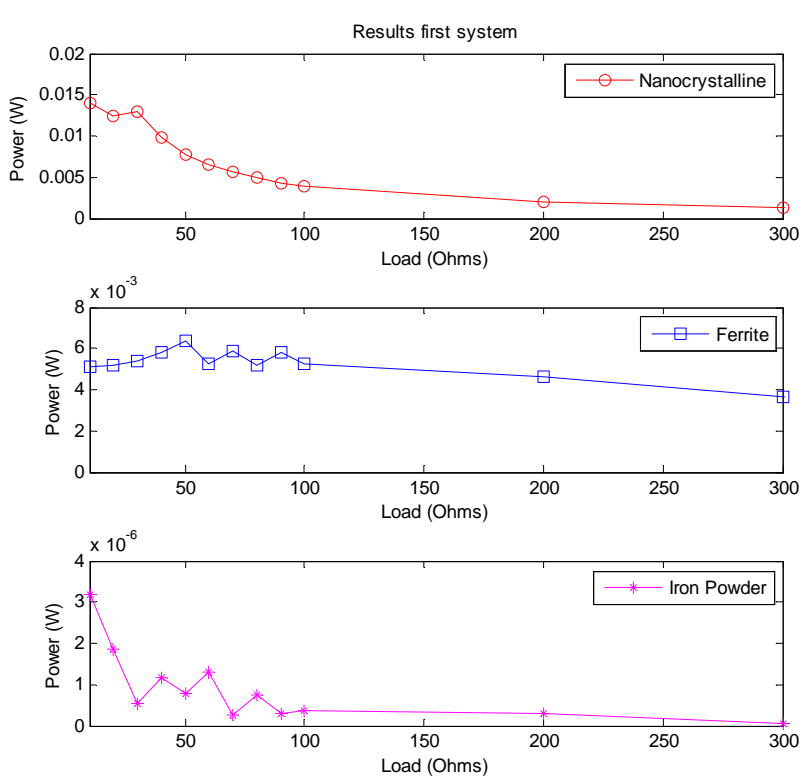

Figure 5. Power levels - first system. 

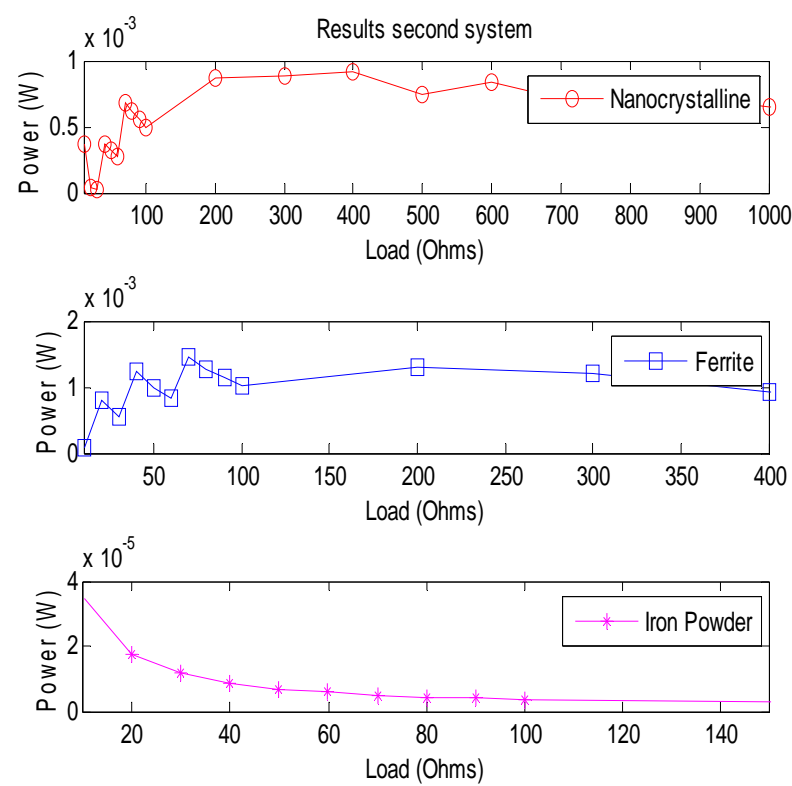

Figure 6. Power levels - second system.

\section{Conclusions}

In this work, it was presented two proposed MagneticDispersion based Energy Harvesting Systems, called 3F-MD-EHS and 1F-MD-EHS, where the former works on three phase conductors and the latter on one phase conductor. Both are based on magnetic cores of different material, named, nanocrystalline, ferrite and iron powder. The obtained experimental results have shown that, considering the 3F-MD-EHS, it was capable of harvesting up to $14 \mathrm{~mW}$ at a load of $10 \Omega$ for a nanocrystalline core with 3 A RMS current on the power line; up to $6.3 \mathrm{~mW}$ at a load of $50 \Omega$ for a ferrite cores with 9 A RMS current on the power line; and up to $3.2 \mu \mathrm{W}$ at a load of $10 \Omega$ for a core of iron powder with 12 A RMS current. Considering the 1F-MD-EHS, it was capable of harvesting up to $0.9 \mathrm{~mW}$ at a load of $400 \Omega$ for the nanocrystalline core with 3 A RMS current on the power line; up to $1.5 \mathrm{~mW}$ at a load of $70 \Omega$ for ferrite cores with 9 A RMS current on the power line, and up to $34 \mu \mathrm{W}$ for a load of $10 \Omega$ for an iron powder core with 12 A current on the power line. In this way, it can be observed that the power harvested from the nanocrystalline core 3F-MD-EHS is 14 times higher than the nanocrystalline core 1F-MD-EHS and 7.5 times higher considering ferrite core. However, considering iron powder cores, the obtained power of the $3 F-M D-$ EHS was 10 times less than in the 1F-MD-EHS.

\section{REFERENCES}

[1] D. M. Toma, J. Del Rio and A. M. Lázaro, "Self-Powered High-Rate Wireless Sensor Network for Underground High Voltage Power Lines," Instrumentation and Measurement Technology Conference, p. c1, 2012.

[2] X. Zhao, M. Ketuel, M. Baldauf and O. Kanaoun, "Energy harvesting for overhead power line monitoring," Systems, Signals and Devices (SSD), International Multi-Conference on, 2012, pp. 1-5.

[3] K. Tashiro, H. Wakiwaka, S. Inoue and Y. Uchiyama, "Energy Harvesting of Magnetic Power-Line Noise," IEEE Transactions on Magnetics, Vol. 47, No.10, 2011 , pp. 4441-4444.

doi.org/10.1109/TMAG.2011.2158190

[4] F. Guo, H. Hayat and J. Wang, "Energy Harvesting Devices for High Voltage Transmission Line Monitoring," Power and Energy Society General Meeting, IEEE, 2011, pp. 1-8. 